\title{
MXene Tunable Lamellae Architectures for
}

\section{Supercapacitor Electrodes}

Vildan Bayram ${ }^{\dagger}$, Michael Ghidiu ${ }^{\S}$, Jae J. Byun ${ }^{\dagger}$, Shelley D. Rawson ${ }^{\ddagger}$, Pei Yang ${ }^{\dagger}$, Samuel A. Mcdonald $^{\star}$, Matthew Lindley ${ }^{\dagger}$, Simon Fairclough ${ }^{\dagger}$, Sarah J. Haigh ${ }^{\dagger}$, Philip J. Withers ${ }^{\dagger, \star}$, Michel W. Barsoum $^{\S}$, Ian A. Kinloch ${ }^{\dagger}$, and Suelen Barg ${ }^{*, \dagger}$

Department of Materials and ${ }^{\dagger}$ The Henry Royce Institute, Department of Materials, University of Manchester, Manchester M13 9PL, U.K.

${ }_{\text {\$Department of Materials Science and Engineering and A. J. Drexel Nanomaterials Institute, Drexel }}$ University, Philadelphia, Pennsylvania 19104, United States

"European Synchrotron Radiation Facility (ESRF), 71 Avenue des Martyrs, 38000 Grenoble, France

*E-mail: Suelen.barg@manchester.ac.uk 

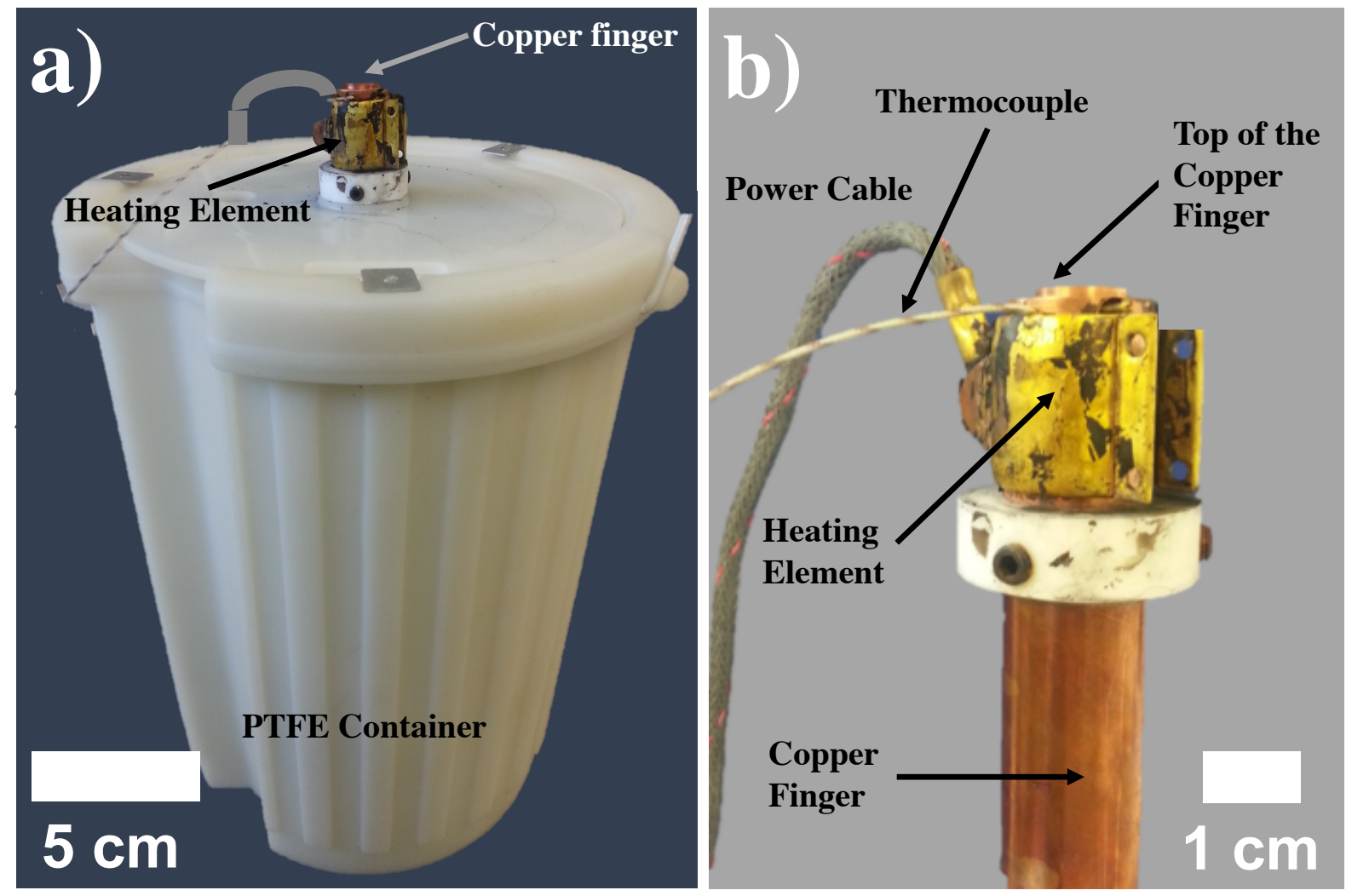

Figure S1. The lab-built freeze caster. a) Outside view of the freeze-caster showing the insulating polytetrafluoroethylene (PTFE) container and the top of the copper cold finger; b) Extended image of the copper cold finger with other components indicated. Prior to freezing, the top temperature of the copper finger is adjusted to room temperature. Freezing parameters are controlled with a thermo-controller that is linked to the heating element and thermo-couple attached to the copper finger. The copper finger temperature directs the freezing of suspensions (cast on moulds and positioned on top of the cold finger). 

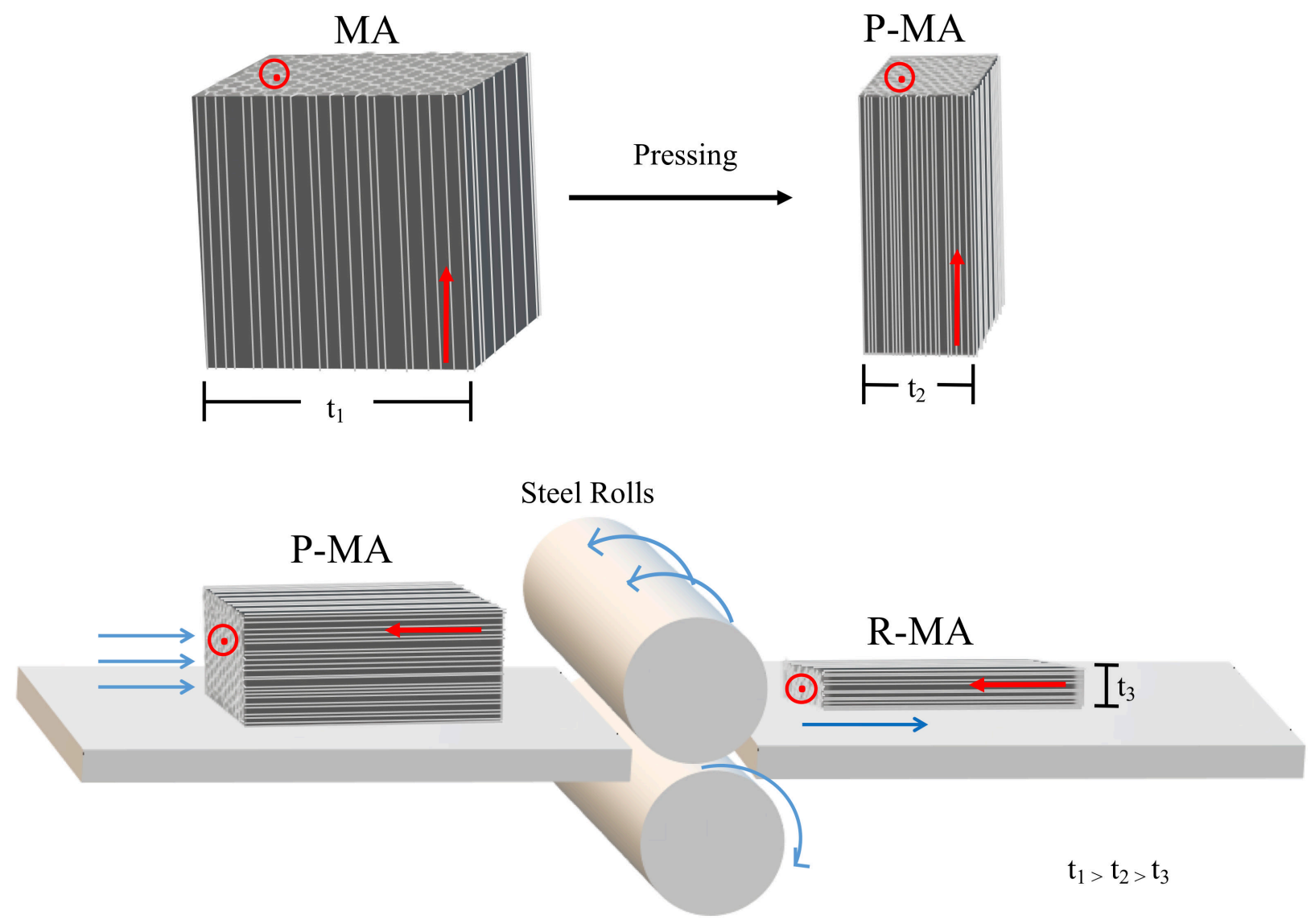

Figure S2. Diagram of pressing of MAs into pressed $\mathrm{Ti}_{3} \mathrm{C}_{2} \mathrm{~T}_{\mathrm{x}}$ aerogels (P-MAs) and the rolling process via calendaring machine demonstrating the propagation of P-MA (through the calendering machine containing two rolls) into a thinner and a more integrated form (transition from P-MA to rolled $\mathrm{Ti}_{3} \mathrm{C}_{2} \mathrm{~T}_{\mathrm{x}}$ aerogels (R-MA). The red arrows and dots on samples show the direction of freezing. Blue arrows indicate the directions of movement. The thicknesses of samples were displayed by $t_{1}, t_{2}$ and $t_{3}$ corresponding to thickness of MA, P-MA and R-MA respectively. 


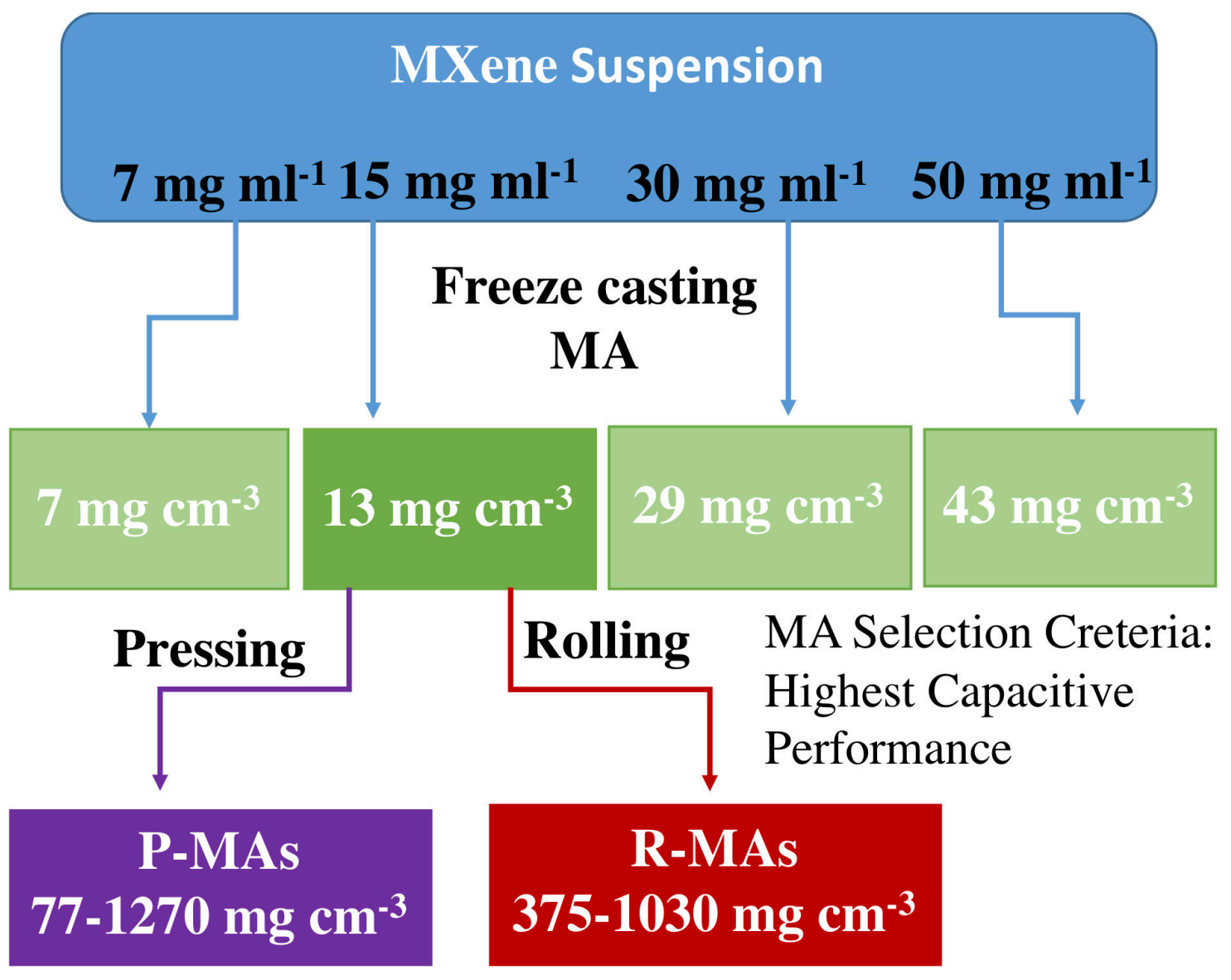

Figure S3. The logical path for electrode fabrication. The MAs with the highest capacitive performance among the MAs with different concentrations were chosen as promising electrode materials for further processes: pressing and rolling with calendaring machine. P-MAs (Pressed MAs) and R-MAs (Rolled MAs) were fabricated with volumetric densities in between 77-1270 $\mathrm{mg} \mathrm{cm}{ }^{-3}$ and $375-1030 \mathrm{mg} \mathrm{cm}^{-3}$, respectively. 

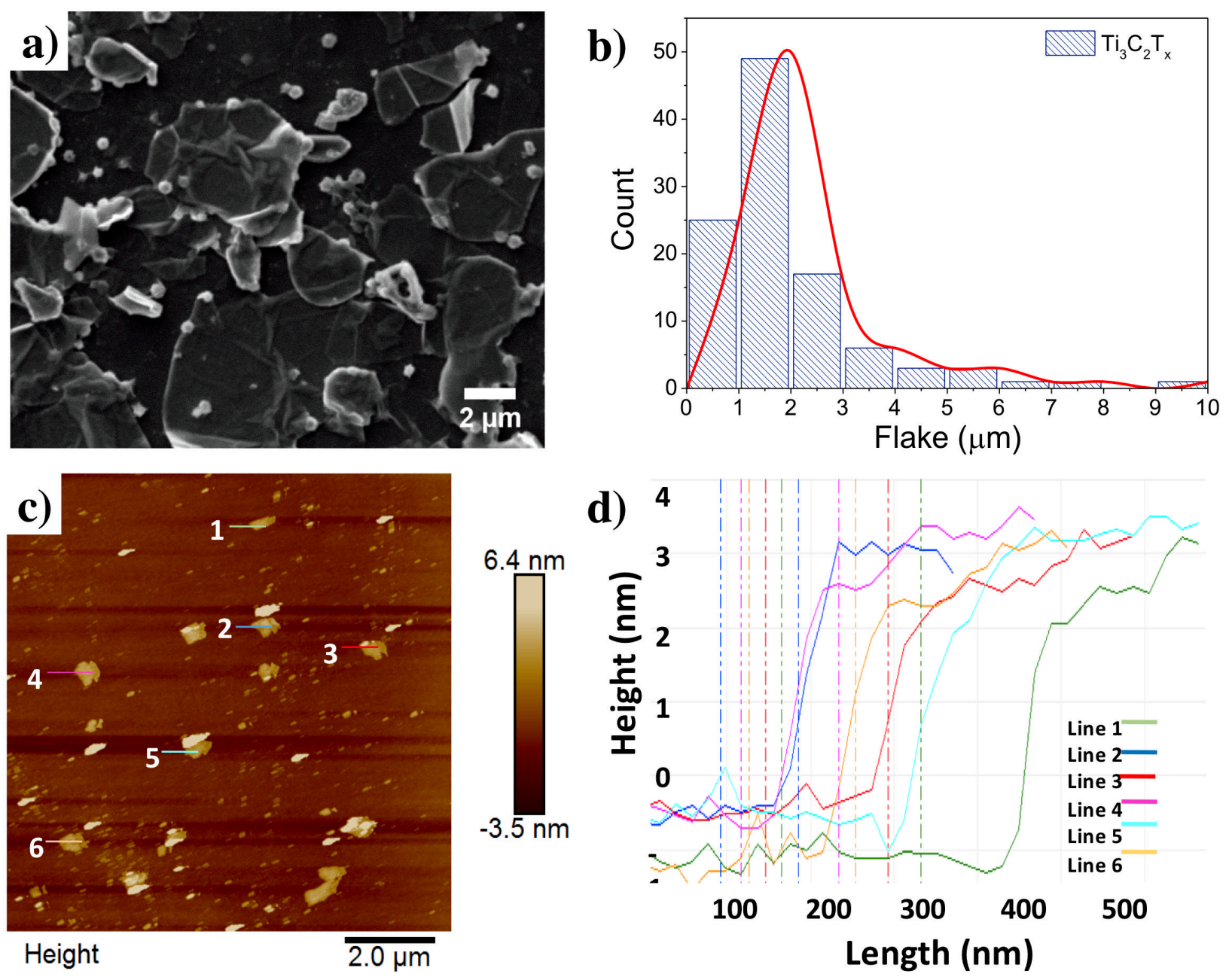

Figure S4. Dimensional analysis of $\mathrm{Ti}_{3} \mathrm{C}_{2} \mathrm{~T}_{\mathrm{x}}$ flakes. a) Example of SEM images used to analyse flake size distribution. b) Flake size distribution obtained from measuring 30 flakes. c) AFM images used to analyse thickness of $\mathrm{Ti}_{3} \mathrm{C}_{2} \mathrm{~T}_{\mathrm{x}}$ flakes. d) Height profiles of the different lines marked on c). 



Figure S5. Scanning electron microscopy (SEM) images of as-prepared $\mathrm{Ti}_{3} \mathrm{C}_{2} \mathrm{~T}_{\mathrm{x}}$ aerogels (MAs) from $15 \mathrm{mg} \mathrm{ml}^{-1}$ frozen at $5^{\circ} \mathrm{C} \mathrm{min}^{-1}$ at a) low magnification and b) higher magnification. The images were taken with cross-sectional view (the freezing direction is indicated with the red vectors) and the yellow circles on b) indicate openings within the aerogel walls. 


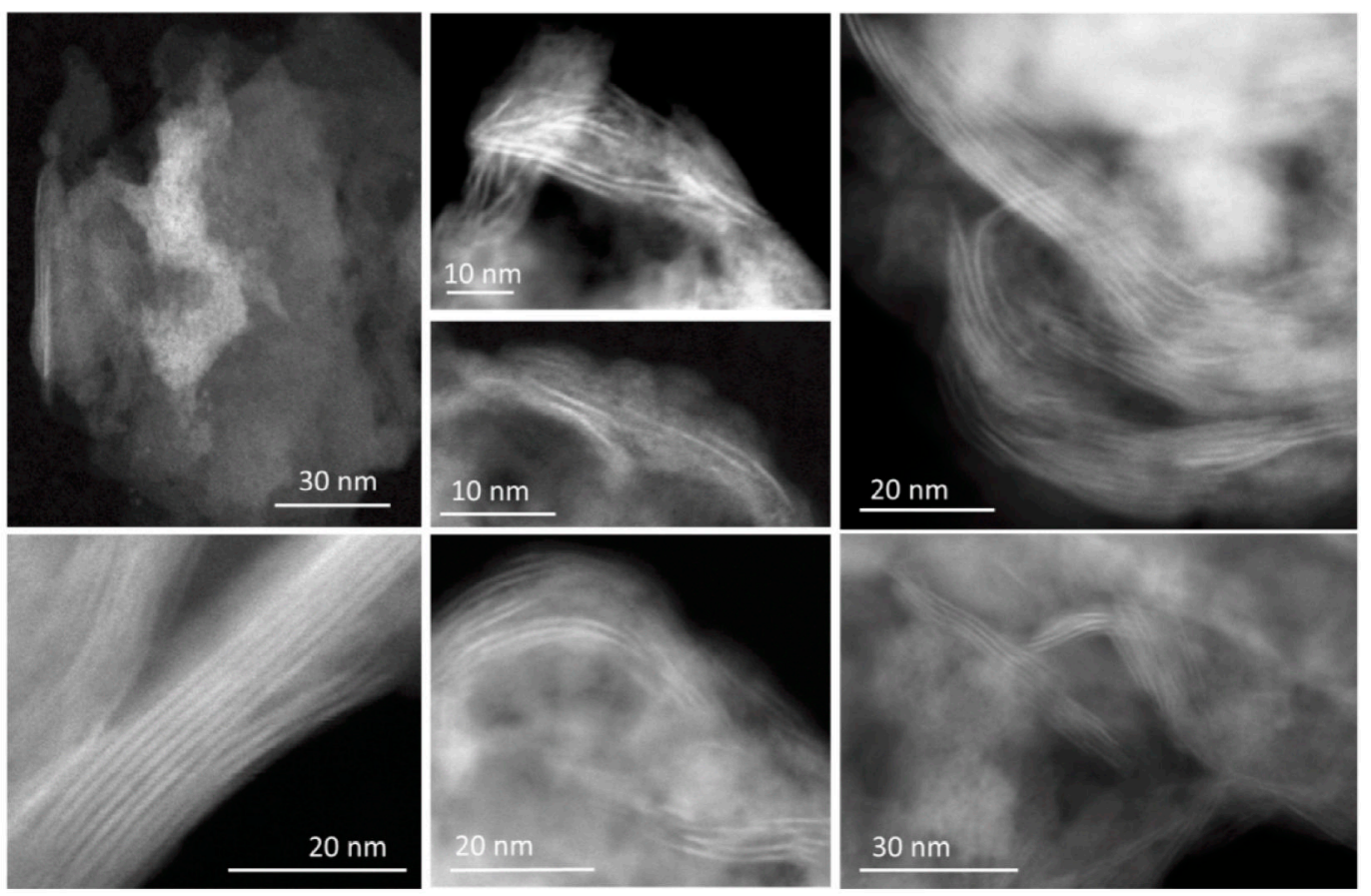

Figure S6. Annular dark field (ADF) scanning transmission electron microscope images (STEM) of $30 \mathrm{mg} \mathrm{ml}^{-1} \mathrm{MA}$ frozen at $5^{\circ} \mathrm{C} \mathrm{min}^{-1}$ freezing rate. Similar images were obtained for the $15 \mathrm{mg}$ $\mathrm{ml}^{-1} \mathrm{MA}$. 


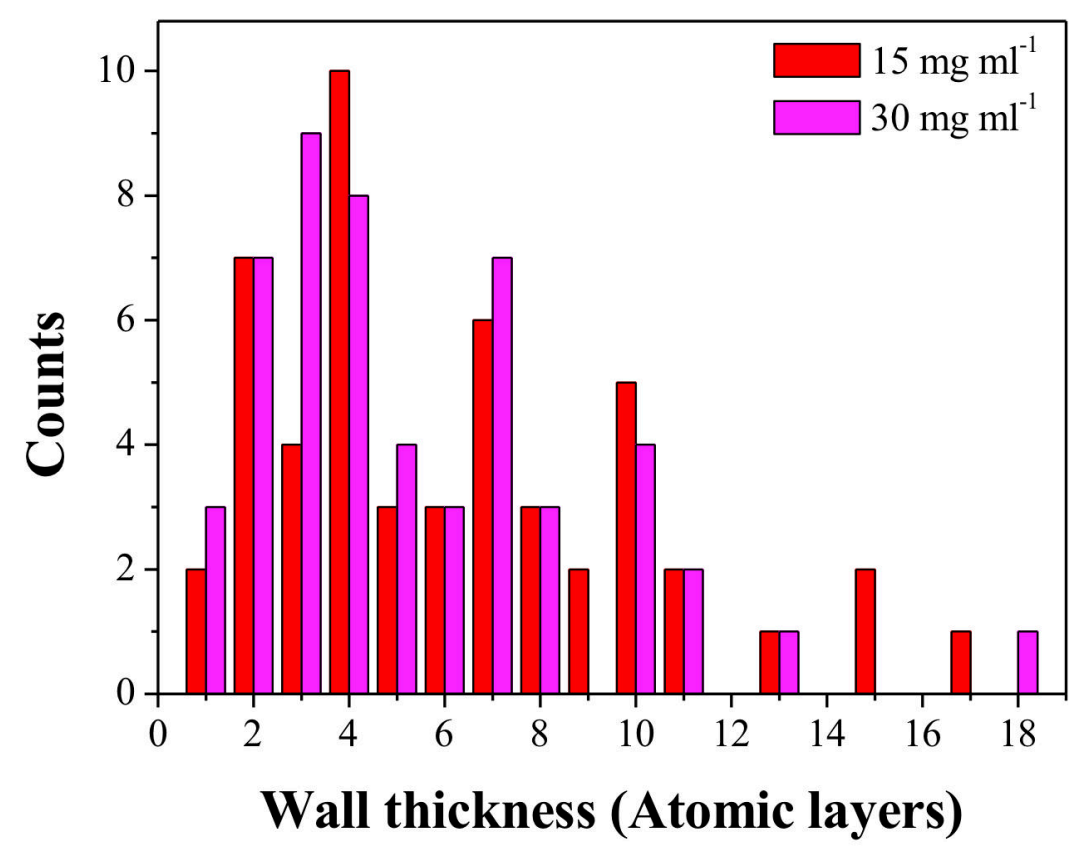

Figure S7. Histograms comparing the wall thicknesses obtained from ADF STEM imaging of 30 $\mathrm{mg} \mathrm{ml} l^{-1}$ and $15 \mathrm{mg} \mathrm{ml}^{-1} \mathrm{MA}$ frozen at $5^{\circ} \mathrm{C} \mathrm{min}^{-1}$ freezing rate. For the $15 \mathrm{mg} \mathrm{ml}^{-1}$ concentration the mean wall thickness from 50 measurements was 6.3 layers with a standard deviation 3.8 layers, For the $30 \mathrm{mg} \mathrm{ml}^{-1}$ concentration the mean wall thickness from 50 measurements was 5.4 layers with a standard deviation 3.5 layers. 

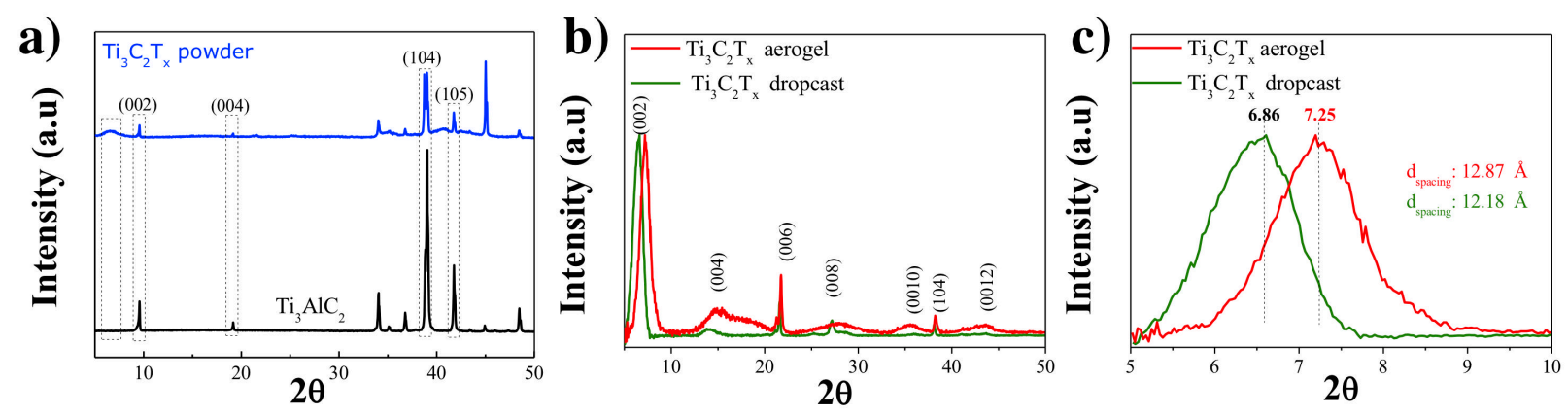

Figure S8. a) XRD of $\mathrm{Ti}_{3} \mathrm{AlC}_{2}$ (black) and $\mathrm{Ti}_{3} \mathrm{C}_{2} \mathrm{~T}_{\mathrm{x}}$ powder (blue) obtained after $\mathrm{LiF}+\mathrm{HCl}$ etching.

b) XRD of $\mathrm{Ti}_{3} \mathrm{C}_{2} \mathrm{~T}_{\mathrm{x}}$ flakes (drop cast) in comparison with $\mathrm{Ti}_{3} \mathrm{C}_{2} \mathrm{~T}_{\mathrm{x}}$ aerogel (MA $-15 \mathrm{mg} \mathrm{ml}^{-1}$ at 2.5

$\left.\left.\mathrm{C} \mathrm{min}^{-1}\right) . \mathrm{c}\right)(002)$ peak zoomed in and d-spacing values are compared. 

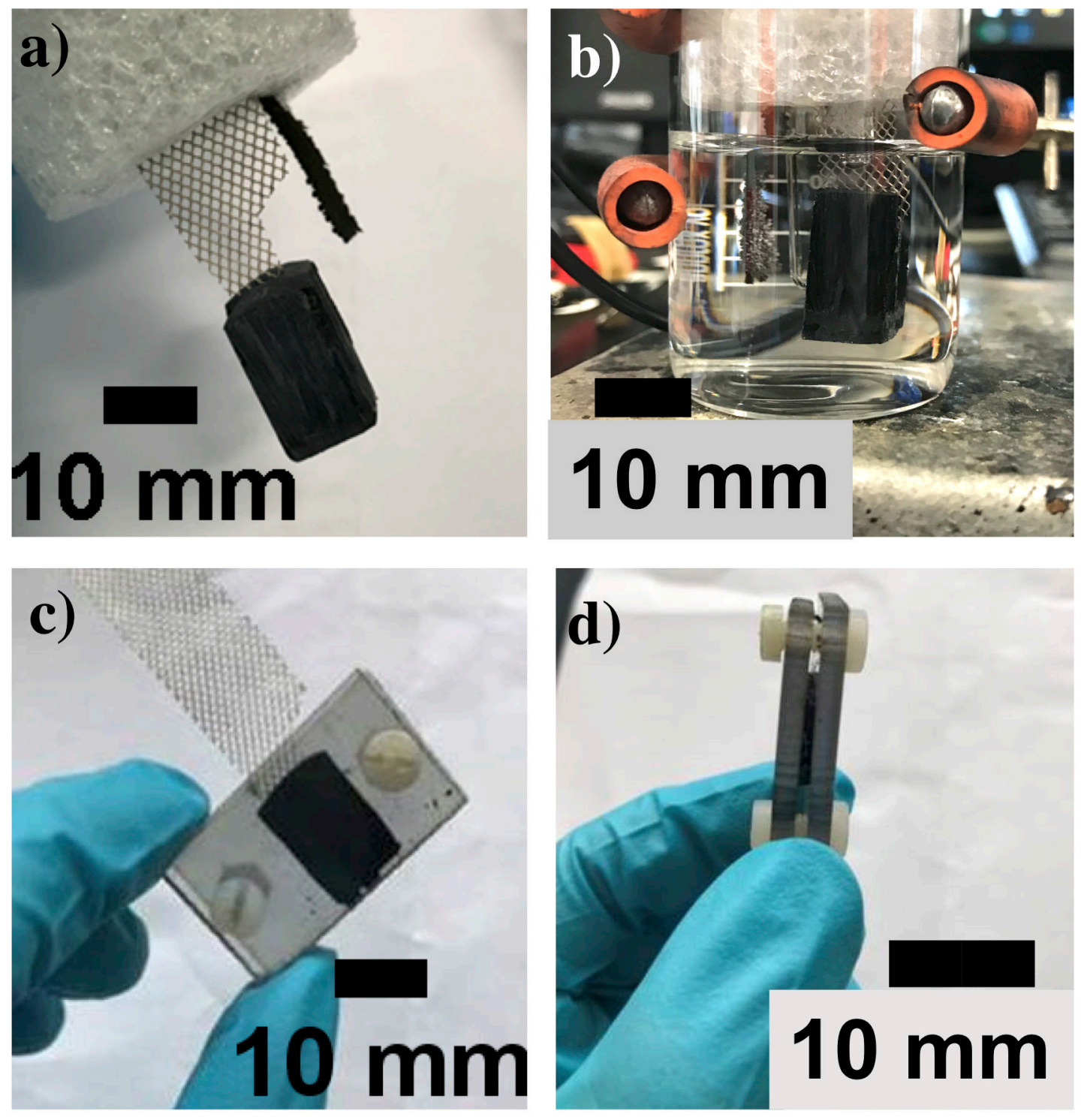

Figure S9. Images for electrochemical testing set-up of $\mathrm{Ti}_{3} \mathrm{C}_{2} \mathrm{~T}_{\mathrm{x}}$ aerogels (MAs). a) $\mathrm{A} \mathrm{Ti}_{3} \mathrm{C}_{2} \mathrm{~T}_{\mathrm{x}}$ aerogel (MA) stabbed with stainless steel current collector. b) $\mathrm{Ti}_{3} \mathrm{C}_{2} \mathrm{~T}_{\mathrm{x}}$ aerogel (MA) immersed into $3 \mathrm{M} \mathrm{H}_{2} \mathrm{SO}_{4}$. c) Pressed $\mathrm{Ti}_{3} \mathrm{C}_{2} \mathrm{~T}_{\mathrm{x}}$ aerogel (P-MA) in between two PMMA slides (side view) d) Bottom view of a pressed $\mathrm{Ti}_{3} \mathrm{C}_{2} \mathrm{~T}_{\mathrm{x}}$ aerogel (P-MA) showing the electrode thickness. 

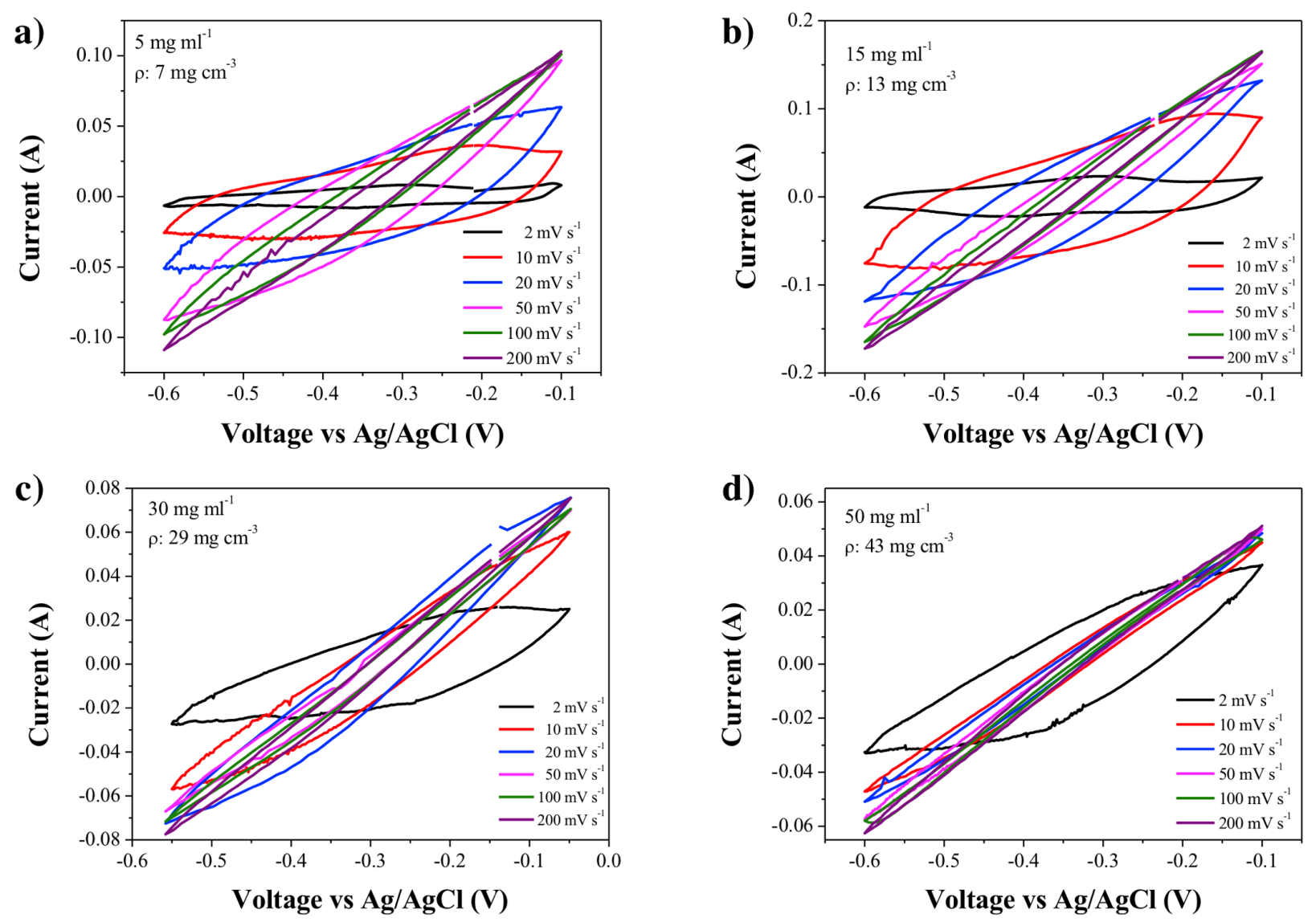

Figure S10. Cyclic Voltammetry (CV) of a) $7 \mathrm{mg} \mathrm{cm}^{-3} \mathrm{MA}$ (produced from $5 \mathrm{mg} \mathrm{ml}^{-1}$ ), b) $13 \mathrm{mg}$ $\mathrm{cm}^{-3} \mathrm{MA}$ (produced from $15 \mathrm{mg} \mathrm{ml}^{-1}$ ), c) $29 \mathrm{mg} \mathrm{cm}^{-3} \mathrm{MA}$ (produced from $30 \mathrm{mg} \mathrm{ml}^{-1}$ ) and d) $43 \mathrm{mg}$ $\mathrm{cm}^{-3} \mathrm{MA}$ (produced from $50 \mathrm{mg} \mathrm{ml}^{-1}$ )at various scan rates $\left(2 \mathrm{mV} \mathrm{s}^{-1}\right.$ up to $\left.200 \mathrm{mV} \mathrm{s}^{-1}\right)$. 

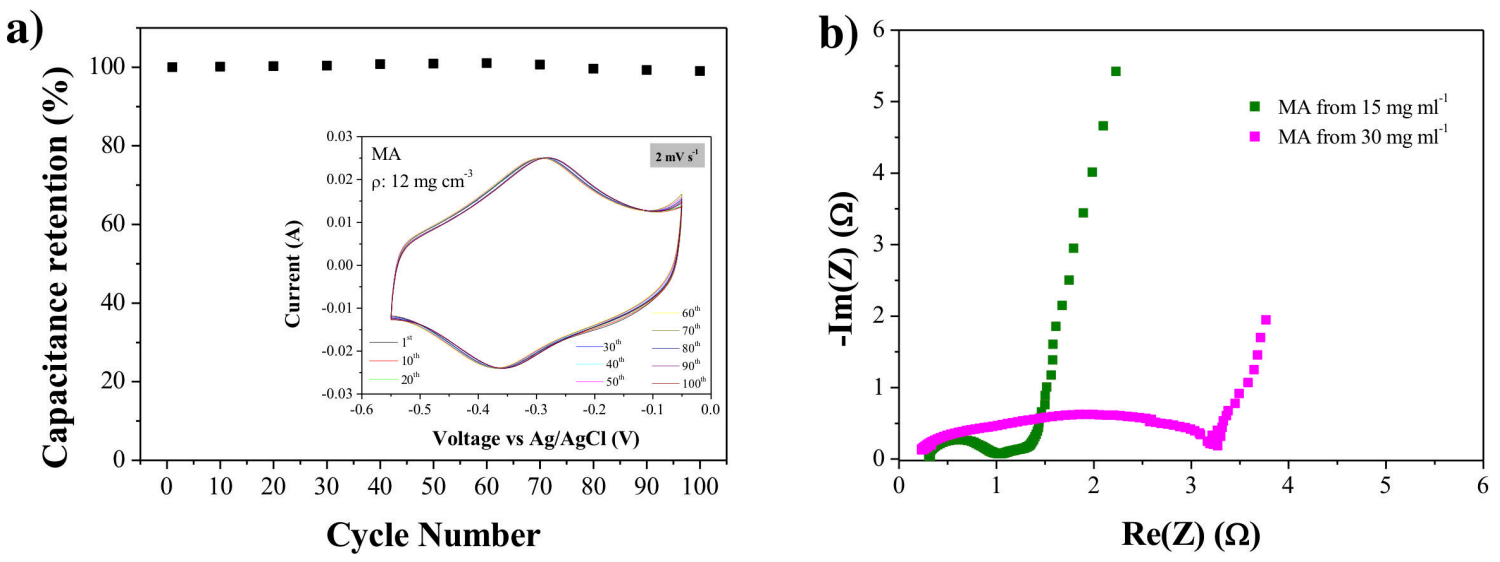

Figure S11. a) Capacitance retention of $12 \mathrm{mg} \mathrm{cm}^{-3} \mathrm{MA}\left(15 \mathrm{mg} \mathrm{ml}^{-1}\right)$ at $2 \mathrm{mV} \mathrm{s}^{-1}$ for a hundred cycles; the inset shows the CV graphs obtained at every ten cycles throughout the cycling test. b) Electrochemical impedance spectroscopy (EIS) of $12 \mathrm{mg} \mathrm{cm}^{-3} \mathrm{MA}$ and $23 \mathrm{mg} \mathrm{cm}^{-3} \mathrm{MA}$ (30 mg $\mathrm{ml}^{-1}$ suspension) collected in the $100 \mathrm{mHz}$ to $100 \mathrm{kHz}$ range using a potential amplitude of $10 \mathrm{mV}$. 


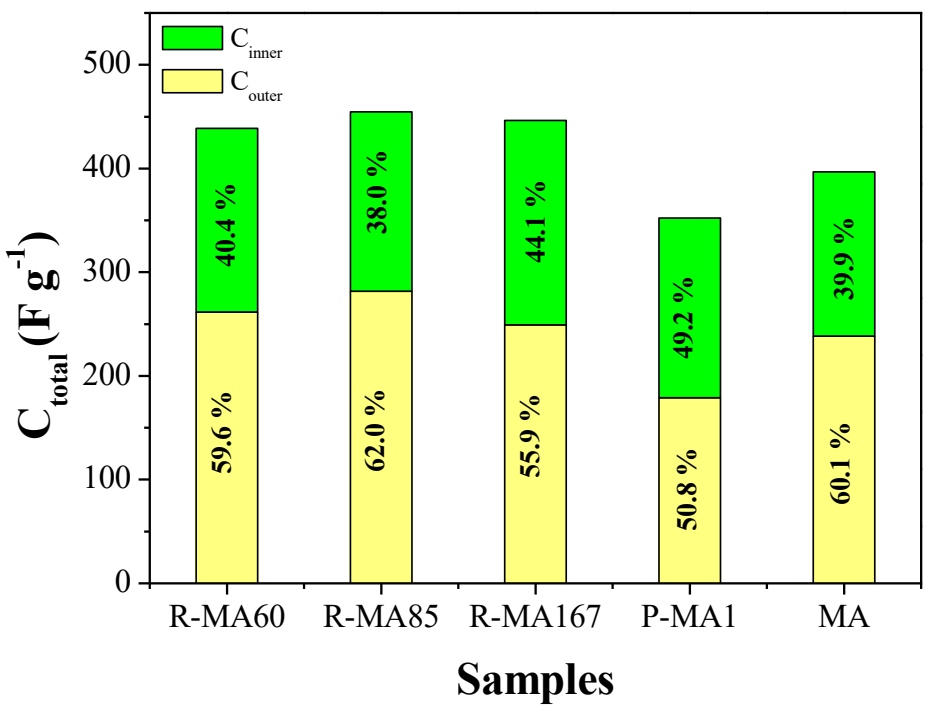

Figure S12. The deconvolution of the capacitance contribution by inner and outer surface of the electrode by Trasatti method ${ }^{1,2}$ in as-prepared $\mathrm{Ti}_{3} \mathrm{C}_{2} \mathrm{~T}_{\mathrm{x}}$ aerogels (MAs), pressed $\mathrm{Ti}_{3} \mathrm{C}_{2} \mathrm{~T}_{\mathrm{x}}$ aerogels (P-MAs) and rolled $\mathrm{Ti}_{3} \mathrm{C}_{2} \mathrm{~T}_{\mathrm{x}}$ aerogels (R-MAs). The percentage of contributions from each process was given on the related bars (green colour used for inner capacitance contributions due to the diffusion-controlled processes and yellow colour used for non-diffusion-limited processes that includes the charge storage on the outer surface (EDLC and surface redox reactions)). Samples were labelled as R-MA60 ( $\rho: 1030$ mg cm${ }^{-3}$ ), R-MA85 ( $\rho: 794 \mathrm{mg} \mathrm{cm}^{-3}$ ), R-MA167 ( $\rho: 576 \mathrm{mg}$ $\left.\mathrm{cm}^{-3}\right)$, P-MA1 ( $\left.\rho: 77 \mathrm{mg} \mathrm{cm}^{-3}\right)$, MA ( $\left.\rho: 12 \mathrm{mg} \mathrm{cm}^{-3}\right)$. 

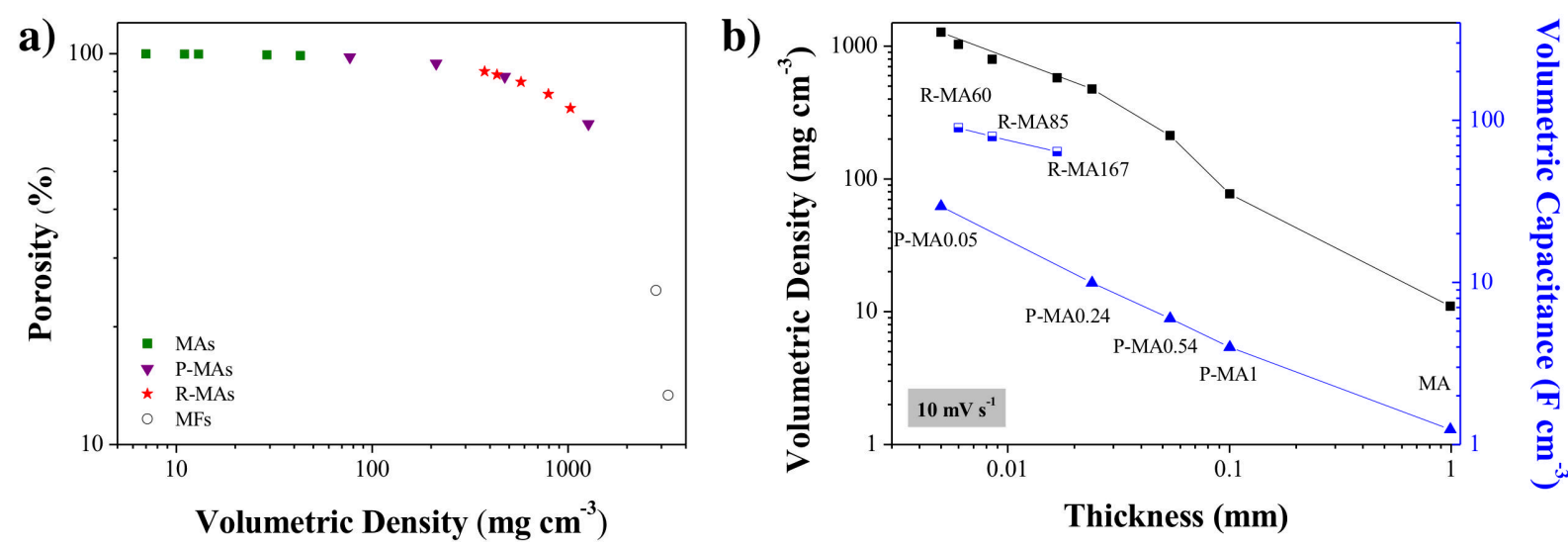

Figure S13. The relationships between a) volumetric density and porosity of electrode materials, and b) volumetric capacitance, volumetric density and thickness of electrode materials; asprepared $\mathrm{Ti}_{3} \mathrm{C}_{2} \mathrm{~T}_{\mathrm{x}}$ aerogels (MAs), pressed $\mathrm{Ti}_{3} \mathrm{C}_{2} \mathrm{~T}_{\mathrm{x}}$ aerogels (P-MAs) and rolled $\mathrm{Ti}_{3} \mathrm{C}_{2} \mathrm{~T}_{\mathrm{x}}$ aerogels (R-MAs). R-MA is produced by rolling a MA. The thickness of the electrode is given by the number following the letters P-MA (in millimetres) and R-MA (in micrometres). 

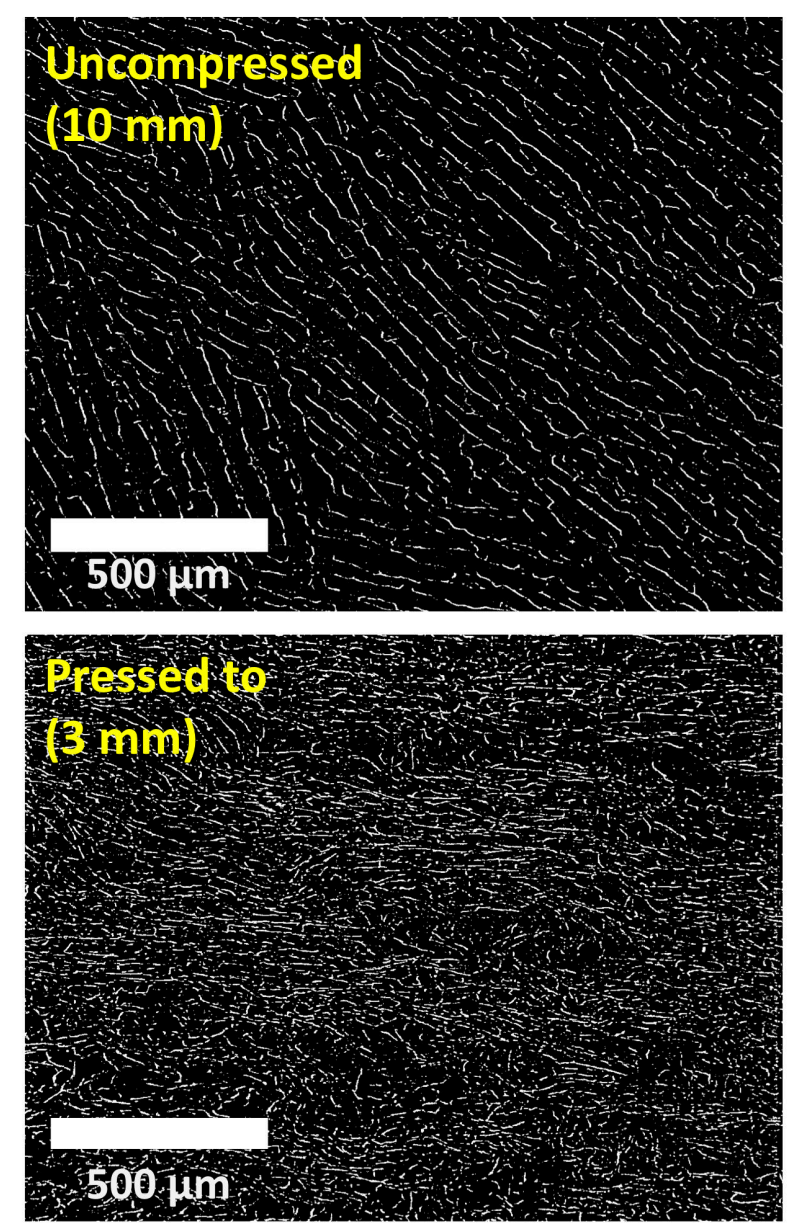
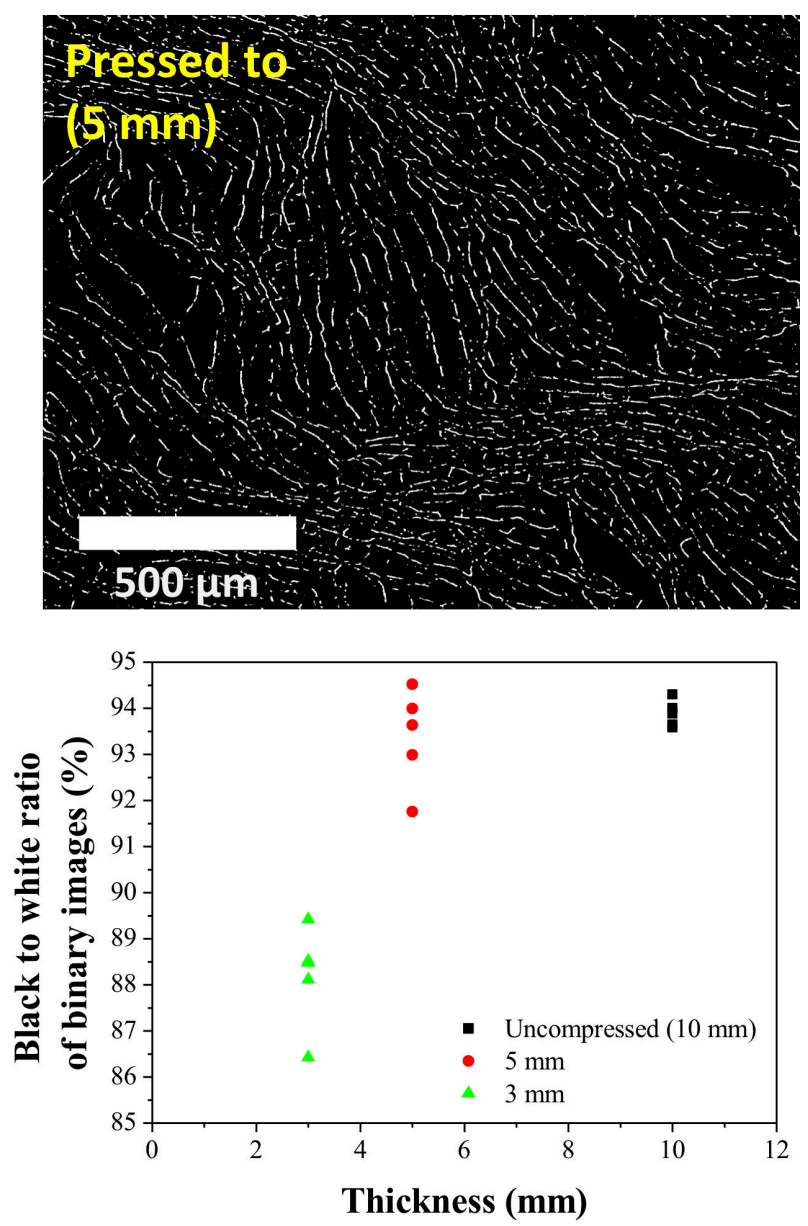

Figure S14. Binary images obtained from Micro-CT (micro computed tomography) images. A volume of sample was scanned with Micro-CT at three different stages; uncompressed (initial thickness $10 \mathrm{~mm}$ ), compressed down to $5 \mathrm{~mm}$ and compressed down to $3 \mathrm{~mm}$, from top to bottom respectively. The ratio of white regions (the lamellae walls) to black regions (the empty space between the lamellae) was plotted with respect to thickness of sample. 

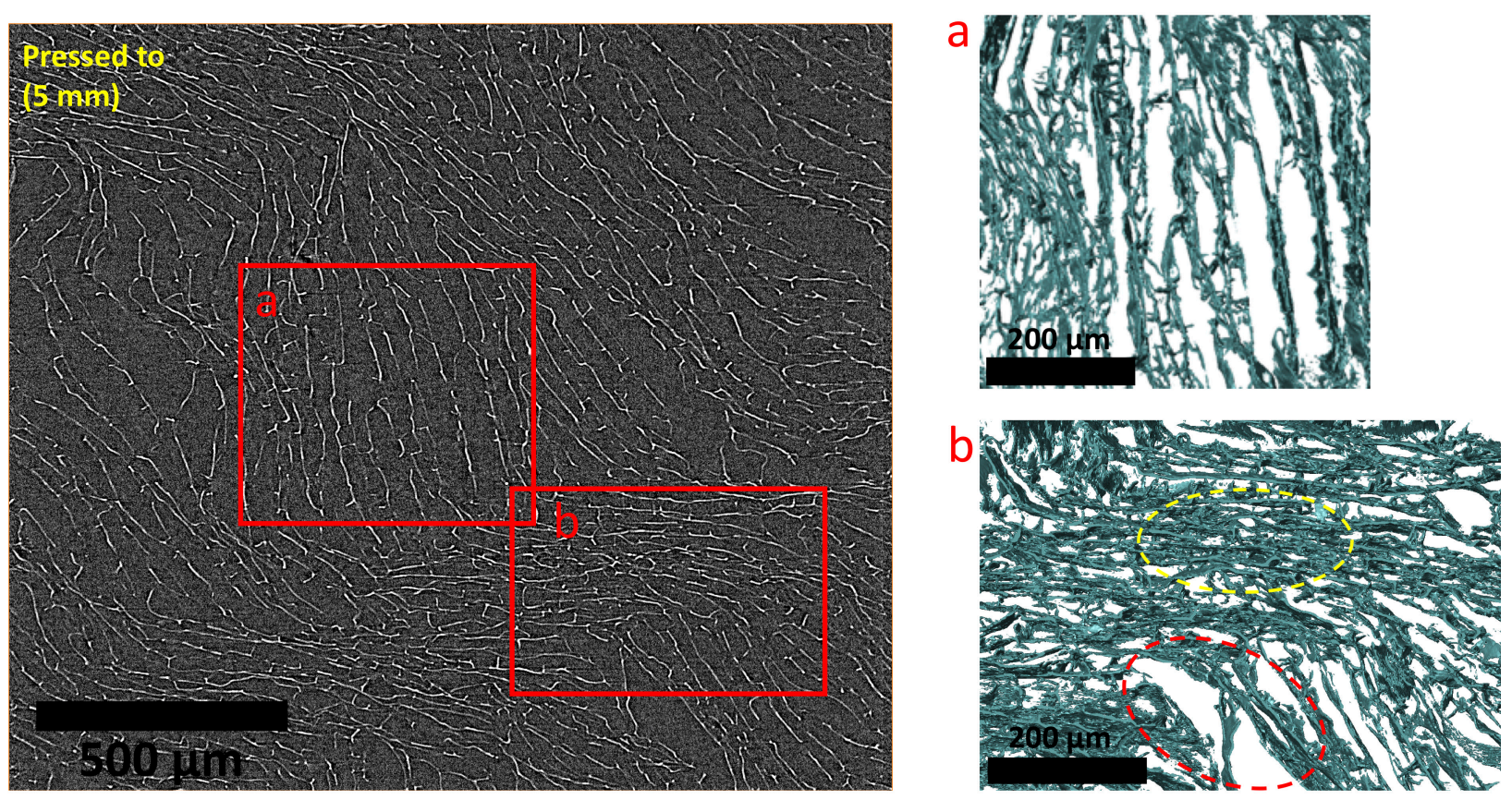

Figure S15. Micro-CT (micro computed tomography) images of P-MA with $5 \mathrm{~mm}$ thickness. Two areas (labelled as $\mathbf{a}$ and $\mathbf{b}$ ) indicated by red squares are visualised for a better understanding of compression mechanism. a possesses a more open structure and $\mathbf{b}$ possesses different regions including crumbled lamellae (indicated by yellow dotted lines) and a wider gap (indicated by red dotted line) upon pressing of MA. 
a)

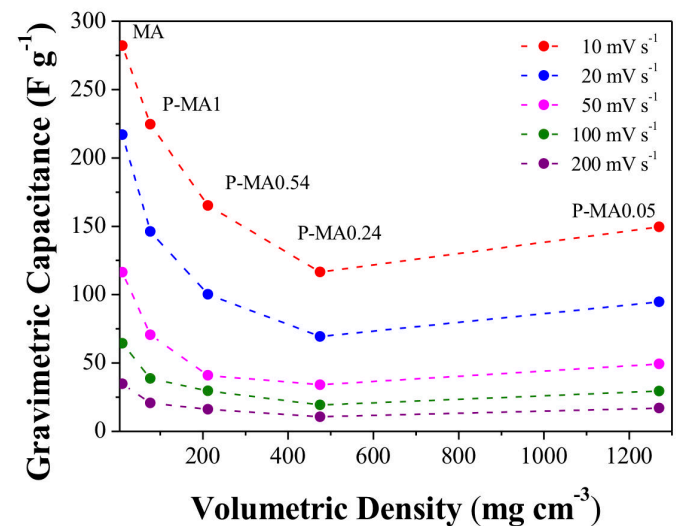

c)

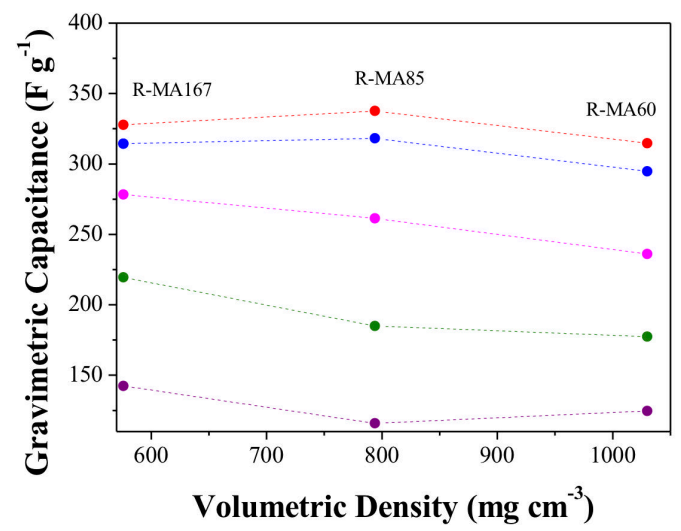

b)

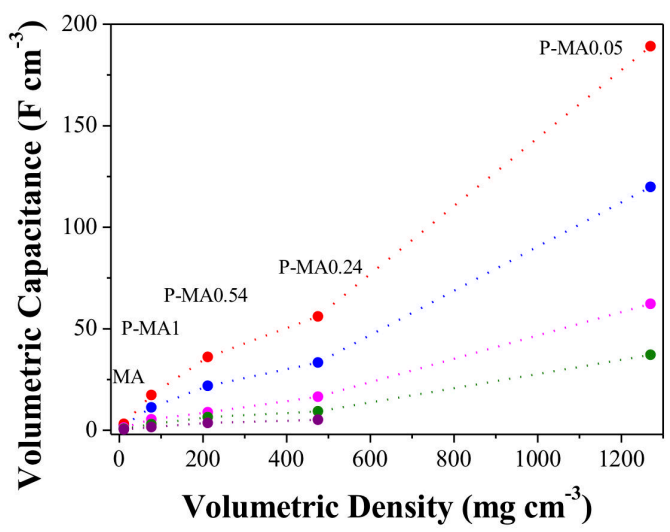

d)

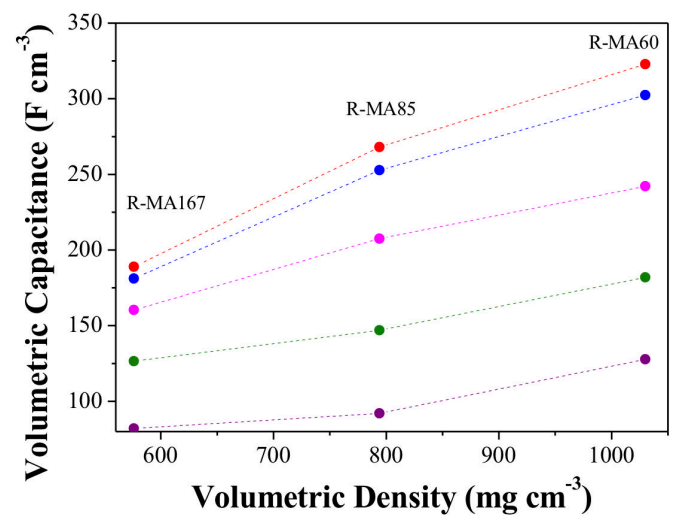

Figure S16. Comparison of a) gravimetric capacitance values and b) volumetric capacitance values for pressed $\mathrm{Ti}_{3} \mathrm{C}_{2} \mathrm{~T}_{\mathrm{x}}$ aerogels (P-MAs) at $10 \mathrm{mV} \mathrm{s}^{-1}, 20 \mathrm{mV} \mathrm{s}^{-1}, 50 \mathrm{mV} \mathrm{s}^{-1}, 100 \mathrm{mV} \mathrm{s}^{-1}$ and $200 \mathrm{mV} \mathrm{s}^{-1}$ with respect to their volumetric densities. Comparison of c) gravimetric capacitance values and d) volumetric capacitance values for rolled $\mathrm{Ti}_{3} \mathrm{C}_{2} \mathrm{~T}_{\mathrm{x}}$ aerogels (R-MAs) at $10 \mathrm{mV} \mathrm{s}{ }^{-1}, 20$ $\mathrm{mV} \mathrm{s}^{-1}, 50 \mathrm{mV} \mathrm{s}^{-1}, 100 \mathrm{mV} \mathrm{s}^{-1}$ with respect to their volumetric densities. The thickness of the electrode is given by the number following the letters with P-MA (in millimetres, mm) and R-MA (in micrometres, $\mu \mathrm{m}$ ) so P-MA1 indicates a P-MA electrode with a thickness of $1 \mathrm{~mm}$. 



Figure S17. Cyclic Voltammetry profiles of pressed (P-MAs) and rolled (R-MAs) $\mathrm{Ti}_{3} \mathrm{C}_{2} \mathrm{~T}_{\mathrm{x}}$ aerogels at various scan rates $\left(2 \mathrm{mV} \mathrm{s}^{-1}\right.$ up to $\left.200 \mathrm{mV} \mathrm{s}^{-1}\right)$. a) $1 \mathrm{~mm}$ thick P-MA. b) $0.54 \mathrm{~mm}$ thick P-MA. c) $0.24 \mathrm{~mm}$ thick P-MA.d) $50 \mu \mathrm{m}$ thick slice rolled $\mathrm{Ti}_{3} \mathrm{C}_{2} \mathrm{~T}_{\mathrm{x}}$ aerogel (SR-MA). e) $167 \mu \mathrm{m}$ thick R-MA. f) $85 \mu \mathrm{m}$ thick R-MA. The densities $(\rho)$ of each sample are indicated inside the graph. 
Pressed MXene Aerogel (P-MA)

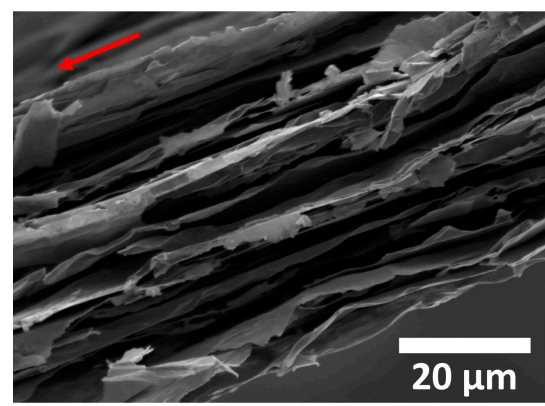

Slice Rolled MXene Aerogel (SR-MA)

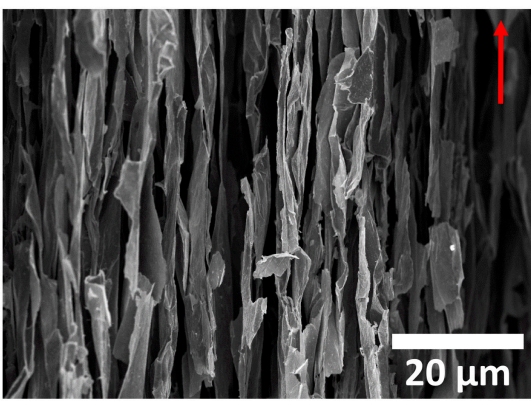

Rolled MXene Aerogel (R-MA)

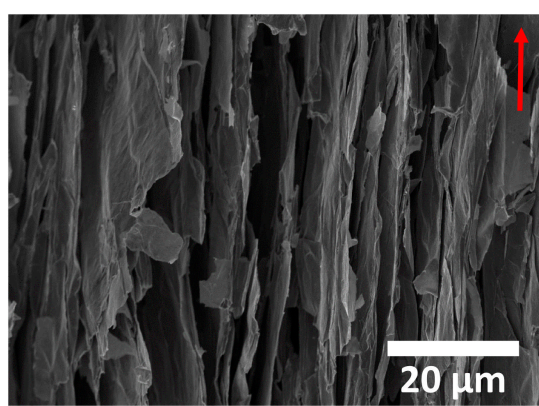

Figure S18. Scanning electron microscopy (SEM) images of pressed $\mathrm{Ti}_{3} \mathrm{C}_{2} \mathrm{~T}_{\mathrm{x}}$ aerogels (P-MAs), slice rolled $\mathrm{Ti}_{3} \mathrm{C}_{2} \mathrm{~T}_{\mathrm{x}}$ aerogels (SR-MA) and rolled $\mathrm{Ti}_{3} \mathrm{C}_{2} \mathrm{~T}_{\mathrm{x}}$ aerogels (R-MA). Images were taken at different orientations (The freezing direction is indicated by the red inserted vectors).
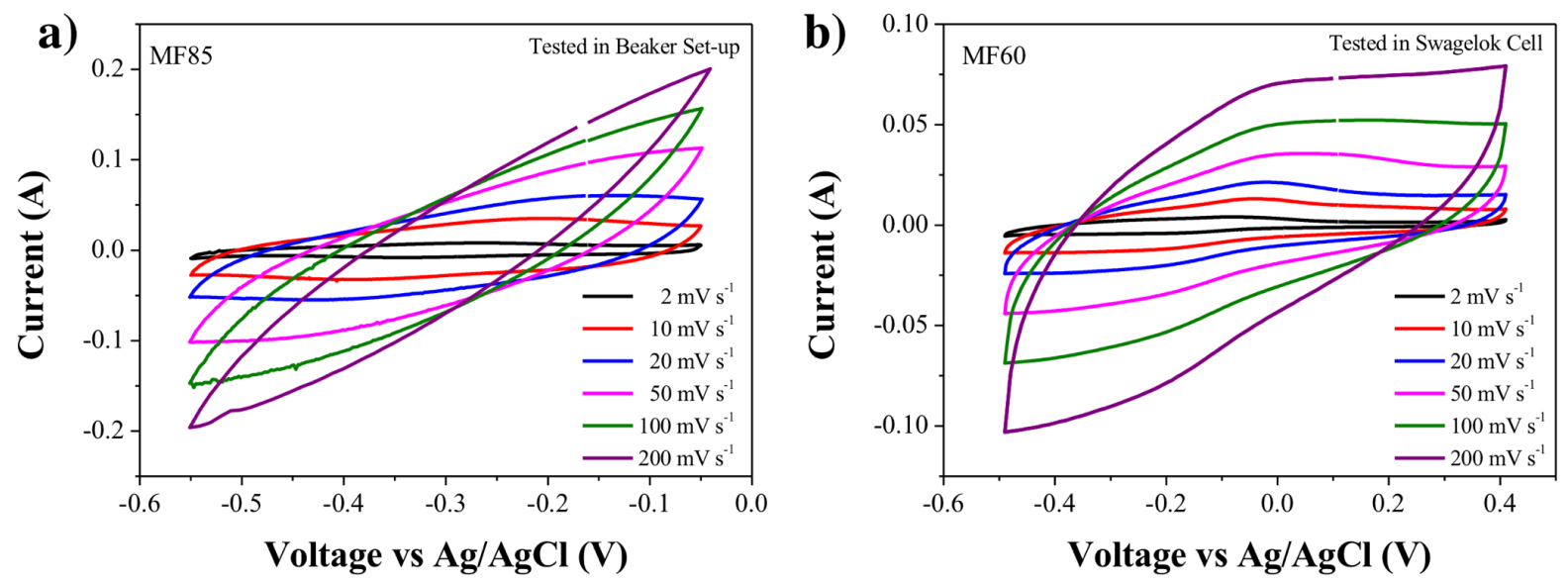

Figure S19. a) MF85 tested in a beaker type electrochemical set-up; b) MF60 filtered film tested in a Swagelok cell. MF stands for $\mathrm{Ti}_{3} \mathrm{C}_{2} \mathrm{~T}_{\mathrm{x}}$ filtered film and number refers to the thickness of the film in $\mu \mathrm{m}$ (MF85 is $85 \mu \mathrm{m}$ thick). 
Table S1. Details of suspension formulations and freezing conditions used to manufacture MXene aerogels (MAs) tested and investigated within this study. The density of as-prepared samples, the spacing within lamellae and the openings within the aerogel walls were measured with Image $\mathbf{J}$ software and monitored along with the changes in processing conditions. SR-MA and R-MA stands for R-MA produced from a slice of an MA or a whole MA, respectively. P-MA stands for the pressed MA.

\begin{tabular}{|c|c|c|c|c|c|c|c|c|c|}
\hline $\begin{array}{c}\text { Sample } \\
\text { Type }\end{array}$ & $\begin{array}{c}\text { Concentration } \\
\left(\mathrm{mg} \mathrm{ml}^{-1}\right)\end{array}$ & $\begin{array}{c}\text { Freezing } \\
\text { Rate } \\
\left({ }^{0} \mathrm{C} \text { min }^{-1}\right)\end{array}$ & $\begin{array}{c}\text { Temperature } \\
\text { Range }\end{array}$ & $\begin{array}{c}\text { Density } \\
\left(\mathrm{mg} \mathrm{cm}^{-3}\right)\end{array}$ & $\begin{array}{c}\text { Spacing } \\
\text { between } \\
\text { Lamellae } \\
(\mu \mathrm{m})\end{array}$ & $\begin{array}{l}\text { STD } \\
(\mu \mathrm{m})\end{array}$ & $\begin{array}{c}\text { Size of the } \\
\text { openings within } \\
\text { Lamellae }(\mu \mathrm{m})\end{array}$ & $\begin{array}{l}\text { STD } \\
(\mu \mathrm{m})\end{array}$ & $\begin{array}{c}\text { Porosity } \\
(\%)\end{array}$ \\
\hline MA & 5 & 2.5 & $20^{\circ} \mathrm{C}$ to $-60^{\circ} \mathrm{C}$ & 7 & - & - & 46 & 21 & 99.8 \\
\hline MA & 15 & 2.5 & $20^{\circ} \mathrm{C}$ to $-60^{\circ} \mathrm{C}$ & 13 & 53 & 29 & 39 & 18 & 99.7 \\
\hline MA & 30 & 2.5 & $20^{\circ} \mathrm{C}$ to $-60^{\circ} \mathrm{C}$ & 29 & 36 & 13 & 47 & 17 & 99.2 \\
\hline MA & 50 & 2.5 & $20^{\circ} \mathrm{C}$ to $-60^{\circ} \mathrm{C}$ & 43 & 32 & 10 & 28 & 16 & 98.9 \\
\hline MA & 15 & 5 & $20^{\circ} \mathrm{C}$ to $-60^{\circ} \mathrm{C}$ & 12 & 51 & 14 & 27 & 11 & 99.7 \\
\hline MA & 30 & 5 & $20^{\circ} \mathrm{C}$ to $-60^{\circ} \mathrm{C}$ & 23 & 41 & 18 & - & - & 99.4 \\
\hline R-MA83 & 15 & 5 & $20^{\circ} \mathrm{C}$ to $-60^{\circ} \mathrm{C}$ & 716 & 1.1 & 0.6 & - & - & 79.4 \\
\hline SR-MA71 & 15 & 5 & $20^{\circ} \mathrm{C}$ to $-60^{\circ} \mathrm{C}$ & 612 & 1.6 & 0.9 & - & - & 83.7 \\
\hline P-MA81 & 15 & 5 & $20^{\circ} \mathrm{C}$ to $-60^{\circ} \mathrm{C}$ & 773 & 1.6 & 0.8 & - & - & 80.9 \\
\hline
\end{tabular}


Table S2. Data used to analyse the electrochemical properties of as-prepared (MA), pressed (PMA) and rolled $\mathrm{Ti}_{3} \mathrm{C}_{2} \mathrm{~T}_{\mathrm{x}}$ aerogels (R-MAs). SR-MA and R-MA stands for R-MA produced from a slice of an MA or a whole MA, respectively. The results are compared with MXene Film (MF) produced via vacuum filtration.

\begin{tabular}{|c|c|c|c|c|c|c|c|c|c|}
\hline \multirow[t]{2}{*}{ Sample Name } & \multirow{2}{*}{$\begin{array}{c}\text { Final Thickness } \\
(\mathrm{cm})\end{array}$} & \multirow{2}{*}{$\begin{array}{c}\text { Porosity } \\
(\%)\end{array}$} & \multirow{2}{*}{$\begin{array}{c}\text { Volumetric Density } \\
\left(\mathrm{mg} \mathrm{cm}^{-3}\right)\end{array}$} & \multicolumn{2}{|c|}{$\begin{array}{c}\text { Capacitance at } \\
\qquad 10 \mathrm{mV} \mathrm{s}^{-1}\end{array}$} & \multicolumn{2}{|c|}{$\begin{array}{l}\text { Capacitance at } \\
\qquad 50 \mathrm{mV} \mathrm{s}^{-1}\end{array}$} & \multicolumn{2}{|c|}{$\begin{array}{l}\text { Capacitance at } \\
200 \mathrm{mV} \mathrm{s}^{-1}\end{array}$} \\
\hline & & & & $\mathrm{F} \mathrm{g}^{-1}$ & $F \mathrm{~cm}^{-3}$ & $\mathrm{~F} \mathrm{~g}^{-1}$ & $F$ cm $^{-3}$ & $\mathrm{~F} \mathrm{~g}^{-1}$ & $\mathrm{~F} \mathrm{~cm}^{-3}$ \\
\hline MA & 0.991 & 99.7 & 11 & 282.0 & 3.1 & 116.3 & 1.3 & 34.8 & 0.4 \\
\hline P-MA0.1 & 0.100 & 97.9 & 77 & 224.6 & 17.3 & 70.6 & 5.4 & 39.7 & 1.6 \\
\hline P-MA0.54 & 0.054 & 94.3 & 212 & 165.2 & 36.1 & 40.8 & 8.9 & 16.6 & 3.6 \\
\hline P-MA0.24 & 0.024 & 87.3 & 475 & 116.5 & 56.0 & 34.1 & 16.5 & 10.7 & 5.2 \\
\hline P-MA0.05 & 0.005 & 66.1 & 1270 & 149.5 & 189.1 & 49.3 & 62.3 & 17.1 & 21.6 \\
\hline SR-MA50 & 0.005 & 90.0 & 375 & 331.8 & 124.4 & 286.7 & 107.5 & 259.8 & 97.4 \\
\hline SR-MA60 & 0.006 & 88.4 & 434 & 421.4 & 182.9 & 308.8 & 134.0 & 253.2 & 109.9 \\
\hline R-MA167 & 0.017 & 84.6 & 576 & 327.7 & 188.9 & 278.3 & 160.4 & 142.3 & 82.0 \\
\hline R-MA85 & 0.009 & 78.8 & 794 & 337.6 & 268.1 & 261.4 & 207.6 & 115.9 & 92.0 \\
\hline R-MA60 & 0.006 & 72.5 & 1030 & 314.7 & 322.8 & 236 & 242.1 & 124.5 & 127.7 \\
\hline MF60 & 0.006 & 24.8 & 2819 & 186.3 & 525.2 & 113 & 318.7 & 186.8 & 526.6 \\
\hline MF85 & 0.009 & 13.4 & 3247 & 337.1 & 109.4 & 177.5 & 57.6 & 72.2 & 23.4 \\
\hline
\end{tabular}


Table S3. Table demonstrating both the volumetric and gravimetric capacitance values for asprepared $\mathrm{Ti}_{3} \mathrm{C}_{2} \mathrm{~T}_{\mathrm{x}}$ aerogels (MA) of different densities $(\rho)$, produced from various concentrations ( $5 \mathrm{mg} \mathrm{ml}^{-1}, 15 \mathrm{mg} \mathrm{ml}^{-1}, 30 \mathrm{mg} \mathrm{ml}^{-1}$ and $50 \mathrm{mg} \mathrm{ml}^{-1}$ )

\begin{tabular}{|c|c|c|c|c|c|c|c|c|}
\hline \multirow{2}{*}{ Scan rate $\left(\mathrm{mV} \mathrm{s}^{-1}\right)$} & \multicolumn{2}{|c|}{$\begin{array}{c}5 \mathrm{mg} \mathrm{ml}^{-1} \\
\rho: 7 \mathrm{mg} \mathrm{cm}^{-3}\end{array}$} & \multicolumn{2}{|c|}{$\begin{array}{c}15 \mathrm{mg} \mathrm{ml}^{-1} \\
\rho: 13 \mathrm{mg} \mathrm{cm}^{-3}\end{array}$} & \multicolumn{2}{|c|}{$\begin{array}{c}30 \mathrm{mg} \mathrm{ml}^{-1} \\
\rho: 29 \mathrm{~m} \mathrm{~cm}^{-3}\end{array}$} & \multicolumn{2}{|c|}{$\begin{array}{c}50 \mathrm{mg} \mathrm{ml}^{-1} \\
\rho: 43 \mathrm{mg} \mathrm{cm}^{-3}\end{array}$} \\
\hline & $F \mathbf{g}^{-1}$ & $F \mathbf{c m}^{-3}$ & $\mathrm{~F} \mathrm{~g}^{-1}$ & $F \mathrm{~cm}^{-3}$ & $F g^{-1}$ & $F \mathrm{~cm}^{-3}$ & $F \mathbf{g}^{-1}$ & $\mathrm{~F} \mathrm{~cm}^{-3}$ \\
\hline 2 & 280 & 2 & 380 & 5 & 199 & 5.8 & 145 & 6.2 \\
\hline 10 & 224 & 1.6 & 268 & 3.5 & 79 & 2.2 & 35 & 1.5 \\
\hline 20 & 168 & 1.2 & 170 & 2.2 & 48 & 1.4 & 18 & 0.8 \\
\hline 50 & 94 & 0.7 & 75 & 1 & 18 & 0.5 & 8 & 0.3 \\
\hline 100 & 50 & 0.4 & 41 & 0.5 & 9 & 0.3 & 4 & 0.2 \\
\hline 200 & 27 & 0.2 & 21 & 0.3 & 5 & 0.1 & 2 & 0.1 \\
\hline
\end{tabular}

Table S4. Specific surface area (SSA) of as-prepared $\mathrm{Ti}_{3} \mathrm{C}_{2} \mathrm{~T}_{\mathrm{x}}$ aerogels (MA), pressed $\mathrm{Ti}_{3} \mathrm{C}_{2} \mathrm{~T}_{\mathrm{x}}$ aerogels (P-MAs), slice rolled $\mathrm{Ti}_{3} \mathrm{C}_{2} \mathrm{~T}_{\mathrm{x}}$ aerogels (SR-MA) and rolled $\mathrm{Ti}_{3} \mathrm{C}_{2} \mathrm{~T}_{\mathrm{x}}$ aerogels (R-MA). The numbers at end of sample name corresponds to their thickness after pressing (P-MAs in mm) or after calendering (R-MAs in $\mu \mathrm{m})$.

\begin{tabular}{|c|c|c|c|c|c|}
\hline Sample & $\begin{array}{c}\text { Thickness } \\
\text { (cm) }\end{array}$ & $\begin{array}{c}\text { Density } \\
\left(\mathbf{m g ~ c m}^{-3}\right)\end{array}$ & $\begin{array}{c}\text { Specific } \\
\text { Surface Area } \\
\left(\mathbf{m}^{2} \mathbf{g}^{-1}\right) \\
\end{array}$ & $\begin{array}{l}\text { Average pore } \\
\text { size }(\mathbf{n m})\end{array}$ & $\begin{array}{l}\text { Pore volume } \\
\qquad\left(\mathrm{cc} \mathrm{g}^{-1}\right)\end{array}$ \\
\hline MA & 0.892 & 13 & 30.122 & 1.580 & 0.131 \\
\hline R-MA193 & 0.019 & 468 & 39.831 & 1.580 & 0.414 \\
\hline SR-MA171 & 0.017 & 377 & 31.523 & 1.820 & 0.256 \\
\hline P-MA0.18 & 0.018 & 346 & 30.502 & 1.960 & 0.241 \\
\hline
\end{tabular}


Table S5. The electrochemical performances of porous MXene electrodes reported in the current literature. $\mathrm{C}_{\mathrm{g}}, \mathrm{C}_{\mathrm{v}}$ and $\mathrm{C}_{\mathrm{a}}$ corresponds to gravimetric, volumetric and areal capacitance values. The references of the processing methods for porous MXene structures were given in the table. ${ }^{3-11}$

\begin{tabular}{|c|c|c|c|c|c|c|c|c|c|c|c|}
\hline Sample & Method & $\begin{array}{c}\text { Volumetric } \\
\text { density } \\
\left(\mathrm{mg} \mathrm{cm}^{-3}\right)\end{array}$ & Thickness & Electrolyte & Potential (V) & $\begin{array}{c}\text { Scan } \\
\text { rates } \\
\left(\mathbf{m V ~ s}^{-1}\right)\end{array}$ & $\underset{\left(\mathbf{F ~ g}^{-1}\right)}{\mathbf{C}_{\mathbf{g}}}$ & $\begin{array}{c}\mathrm{C}_{\mathbf{v}} \\
\left(\mathrm{F} \mathrm{cm}^{-3}\right)\end{array}$ & $\begin{array}{c}\mathrm{C}_{\mathrm{a}} \\
\left(\mathrm{mF} \mathrm{cm}^{-2}\right)\end{array}$ & Rate performance & Application \\
\hline $\mathrm{Ti}_{3} \mathrm{C}_{2} \mathrm{~T}_{\mathrm{x}}$ & Clay rolled ${ }^{3}$ & 2200 & $75 \mu \mathrm{m}$ & $1 \mathrm{M} \mathrm{H}_{2} \mathrm{SO}_{4}$ & $\begin{array}{c}0.55(-0.3-0.25) \\
(\mathrm{Ag} / \mathrm{AgCl})\end{array}$ & 50 & 102 & - & - & $\begin{array}{c}100 \% \text { at } 10 \mathrm{~A} \mathrm{~g}^{-1} \\
\text { after } 10000 \text { cycles }\end{array}$ & Supercapacitor \\
\hline $\mathrm{Ti}_{3} \mathrm{C}_{2} \mathrm{~T}_{\mathrm{x}}$ & $\begin{array}{c}\text { Macroporous film by } \\
\text { Sacrificial templating }^{4}\end{array}$ & 240 & $180 \mu \mathrm{m}$ & $3 \mathrm{M} \mathrm{H}_{2} \mathrm{SO}_{4}$ & $\begin{array}{l}1(-1.1--0.1) \\
\left(\mathrm{Hg} / \mathrm{Hg}_{2} \mathrm{SO}_{4}\right)\end{array}$ & 50 & 260 & - & 1.1 & - & Supercapacitor \\
\hline $\mathrm{Ti}_{3} \mathrm{C}_{2} \mathrm{~T}_{\mathrm{x}}$ & $\begin{array}{c}\text { Hydrogel film by } \\
\text { Sacrificial templating }^{4}\end{array}$ & 220 & $40 \mu \mathrm{m}$ & $3 \mathrm{M} \mathrm{H}_{2} \mathrm{SO}_{4}$ & $\begin{array}{l}(-1.1--0.1) \\
\left(\mathrm{Hg} / \mathrm{Hg}_{2} \mathrm{SO}_{4}\right)\end{array}$ & 50 & 331 & 1000 & 2.5 & $\begin{array}{c}90 \% \text { at } 10 \mathrm{~A} \mathrm{~g}^{-1} \\
\text { after } 10000 \text { cycles }\end{array}$ & Supercapacitor \\
\hline $\mathrm{Ti}_{3} \mathrm{C}_{2} \mathrm{~T}_{\mathrm{x}}$ & $\begin{array}{l}\text { Vacuum-assisted } \\
\text { filtration }^{5}\end{array}$ & - & $1.17 \mu \mathrm{m}$ & $3 \mathrm{M} \mathrm{H}_{2} \mathrm{SO}_{4}$ & $\begin{array}{c}1(-0.6-0.4) \\
(\mathrm{Ag} / \mathrm{AgCl})\end{array}$ & 50 & 270 & 1000 & - & $\begin{array}{c}100 \% \text { at } 10 \mathrm{~A} \mathrm{~g}^{-1} \\
\text { after } 10000 \text { cycles }\end{array}$ & Supercapacitor \\
\hline $\begin{array}{c}\mathrm{Ti}_{3} \mathrm{C}_{2} \mathrm{~T}_{\mathrm{x}} \\
\text { Liquid Crystal } \\
\end{array}$ & $\begin{array}{c}\text { Uniaxial in-plane } \\
\text { mechanical shearing }\end{array}$ & 140 & $200 \mu \mathrm{m}$ & $3 \mathrm{M} \mathrm{H}_{2} \mathrm{SO}_{4}$ & $\begin{array}{l}0.9(-1--0.1) \\
\left(\mathrm{Hg} / \mathrm{Hg}_{2} \mathrm{SO}_{4}\right)\end{array}$ & 50 & 270 & - & 0.75 & $\begin{array}{c}100 \% \text { at } 20 \mathrm{~A} \mathrm{~g}^{-1} \\
\text { after } 20000 \text { cycles }\end{array}$ & Supercapacitor \\
\hline $\mathrm{Ti}_{3} \mathrm{C}_{2} \mathrm{~T}_{\mathrm{x}}-\mathrm{rGO}$ & Freeze-drying ${ }^{7}$ & - & - & PVA- $\mathrm{H}_{2} \mathrm{SO}_{4}$ & $0-0.6$ & 1 & - & - & 34.6 & $\begin{array}{l}91 \% \text { at } 2 \mathrm{~mA} \mathrm{~cm}^{-2} \\
\text { after } 15000 \text { cycles }\end{array}$ & Microsupercapacitor \\
\hline $\mathrm{Ti}_{3} \mathrm{C}_{2} \mathrm{~T}_{\mathrm{x}}$ & $\begin{array}{c}\text { EDA-assisted self- } \\
\text { assembly }{ }^{8}\end{array}$ & - & - & $1 \mathrm{M} \mathrm{KOH}$ & $\begin{array}{c}0.6(-1--0.4) \\
(\mathrm{Ag} / \mathrm{AgCl})\end{array}$ & 2 & 87.1 & - & 1012.5 & $\begin{array}{c}95 \% \text { at } 2 \mathrm{~A} \mathrm{~g}^{-1} \text { after } \\
10000 \text { cycles }\end{array}$ & Supercapacitor \\
\hline $\mathrm{Ti}_{3} \mathrm{C}_{2} \mathrm{~T}_{\mathrm{x}}$ & Vacuum freeze-drying ${ }^{9}$ & 2.5 & $4 \mu \mathrm{m}$ & $1 \mathrm{M} \mathrm{NaCl}$ & $\begin{array}{c}0.6(-0.8--0.2) \\
(\mathrm{Ag} / \mathrm{AgCl})\end{array}$ & 5 & 156 & 410 & - & $\begin{array}{c}103 \% \text { at } 100 \mathrm{mV} \mathrm{s}^{-1} \\
\text { after } 5000 \text { cycles }\end{array}$ & $\begin{array}{c}\text { Capacitive } \\
\text { deionization }\end{array}$ \\
\hline $\mathrm{Ti}_{3} \mathrm{C}_{2} \mathrm{~T}_{\mathrm{x}}$ & $\begin{array}{c}\text { Filtration followed by a } \\
\text { hydrazine induced } \\
\text { foaming process }{ }^{10}\end{array}$ & $500-3500$ & $6-60 \mu \mathrm{m}$ & - & - & - & - & - & - & - & EMI shielding \\
\hline $\mathrm{Ti}_{3} \mathrm{C}_{2} \mathrm{~T}_{\mathrm{x}}$ & $\begin{array}{l}\text { Bidirectional freeze- } \\
\text { casting }{ }^{11}\end{array}$ & 11 & $1.5 \mu \mathrm{m}-1 \mathrm{~mm}$ & - & - & - & - & - & - & - & EMI shielding \\
\hline
\end{tabular}




\section{References}

(1) Trasatti, S.; Buzzanca, G. Ruthenium Dioxide: A New Interesting Electrode Material. Solid State Structure and Electrochemical Behaviour. J. Electroanal. Chem. Interfacial Electrochem. 1971, 29 (2), A1-A5. https://doi.org/10.1016/S0022-0728(71)80111-0.

(2) Sankar, K. V.; Selvan, R. K. The Ternary MnFe2O4/Graphene/Polyaniline Hybrid Composite as Negative Electrode for Supercapacitors. J. Power Sources 2015, 275, 399_ 407. https://doi.org/10.1016/j.jpowsour.2014.10.183.

(3) Ghidiu, M.; Lukatskaya, M. R.; Zhao, M.; Gogotsi, Y.; Barsoum, M. W. Conductive TwoDimensional Titanium Carbide 'Clay’ with High Volumetric Capacitance. Nature 2014, 516 (7529), 78-81. https://doi.org/10.1038/nature13970.

(4) Lukatskaya, M. R.; Kota, S.; Lin, Z.; Zhao, M.-Q.; Shpigel, N.; Levi, M. D.; Halim, J.; Taberna, P.-L.; Barsoum, M. W.; Simon, P.; Gogotsi, Y. Ultra-High-Rate Pseudocapacitive Energy Storage in Two-Dimensional Transition Metal Carbides. Nat. Energy 2017, 2 (8), 17105. https://doi.org/10.1038/nenergy.2017.105.

(5) Maleski, K.; Ren, C. E.; Zhao, M. Q.; Anasori, B.; Gogotsi, Y. Size-Dependent Physical and Electrochemical Properties of Two-Dimensional MXene Flakes. ACS Appl. Mater. Interfaces 2018, 10 (29), 24491-24498. https://doi.org/10.1021/acsami.8b04662.

(6) Xia, Y.; Mathis, T. S.; Zhao, M. Q.; Anasori, B.; Dang, A.; Zhou, Z.; Cho, H.; Gogotsi, Y.; Yang, S. Thickness-Independent Capacitance of Vertically Aligned Liquid-Crystalline MXenes. Nature 2018, 557 (7705), 409-412. https://doi.org/10.1038/s41586-018-0109-z.

(7) Yue, Y.; Liu, N.; Ma, Y.; Wang, S.; Liu, W.; Luo, C.; Zhang, H.; Cheng, F.; Rao, J.; Hu, X.; Su, J.; Gao, Y. Highly Self-Healable 3D Microsupercapacitor with MXene-Graphene 
Composite Aerogel. ACS Nano 2018, 12 (5), 4224-4232.

https://doi.org/10.1021/acsnano.7b07528.

(8) Bao, W.; Tang, X.; Guo, X.; Choi, S.; Wang, C.; Gogotsi, Y.; Wang, G. Porous Cryo-

Dried MXene for Efficient Capacitive Deionization. Joule 2018, 2 (4), 778-787.

https://doi.org/10.1016/J.JOULE.2018.02.018.

(9) Li, L.; Zhang, M.; Zhang, X.; Zhang, Z. New Ti3C2 Aerogel as Promising Negative

Electrode Materials for Asymmetric Supercapacitors. J. Power Sources 2017, 364, 234-

241. https://doi.org/10.1016/j.jpowsour.2017.08.029.

(10) Liu, J.; Zhang, H.-B.; Sun, R.; Liu, Y.; Liu, Z.; Zhou, A.; Yu, Z.-Z. Hydrophobic, Flexible, and Lightweight MXene Foams for High-Performance ElectromagneticInterference Shielding. Adv. Mater. 2017, 1702367.

https://doi.org/10.1002/adma.201702367.

(11) Han, M.; Yin, X.; Hantanasirisakul, K.; Li, X.; Iqbal, A.; Hatter, C. B.; Anasori, B.; Koo, C. M.; Torita, T.; Soda, Y.; Zhang, L.; Cheng, L.; Gogotsi, Y. Anisotropic MXene Aerogels with a Mechanically Tunable Ratio of Electromagnetic Wave Reflection to Absorption. Adv. Opt. Mater. 2019. https://doi.org/10.1002/adom.201900267. 